\title{
The Michigan Appropriateness Guide for Intravenous Catheters in Children with Congenital Heart Disease: miniMAGIC-CHD
}

Tanya Perry a , Amanda J Ullman b, c, d, Ranjit Aiyagari e, Stephanie Pitts ${ }^{\text {f, g }}$, Jeffrey P. Jacobs ${ }^{\text {h, }}$ David S. Cooper ${ }^{\text {a }}$

\author{
Affiliations \\ a The Heart Institute, Cincinnati Children's Hospital Medical Center, Department of Pediatrics, \\ University of Cincinnati College of Medicine, Cincinnati, Ohio, USA; \\ ${ }^{\mathrm{b}}$ Alliance for Vascular Access Teaching and Research, Menzies Health Institute Queensland, \\ Griffith University, Queensland, Australia; \\ ${ }^{c}$ School of Nursing and Midwifery, Griffith University, Queensland, Australia;

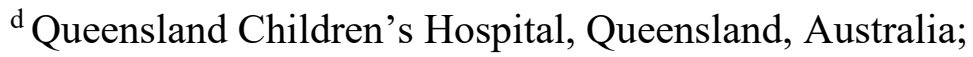 \\ ${ }^{\mathrm{e}}$ Division of Pediatric Cardiology, Department of Pediatrics, University of Michigan Medical \\ School, Ann Arbor, Michigan, USA; \\ ${ }^{\text {f }}$ St Joseph's Children's Hospital, Tampa, Florida. \\ ${ }^{\mathrm{g}}$ B. Braun Medical, Bethlehem, Pennsylvania. \\ ${ }^{\mathrm{h}}$ Congenital Heart Center, Division of Thoracic and Cardiovascular Surgery, Department of \\ Surgery, University of Florida, Gainesville, Florida, USA
}

Key words: congenital heart disease, cardiac critical care, vascular access

\section{Address correspondence to:}

Tanya Perry, DO

The Heart Institute

Cincinnati Children's Hospital Medical Center

3333 Burnet Avenue, MLC 2003

Cincinnati, OH 45229

Telephone: 513-803-9374

E-mail: Tanya.Perry@cchmc.org 


\begin{abstract}
Background

The approach to vascular access in children with paediatric and congenital heart disease is a complex decision-making process that may have long-term implications. To date, evidencebased recommendations have not been established to inform this process.
\end{abstract}

\title{
Methods
}

The RAND/UCLA Appropriateness Method was used to develop miniMAGIC (Michigan Appropriateness Guide for Intravenous Catheters in Pediatrics), including sequential phases: definition of scope and key terms; information synthesis and literature review; expert multidisciplinary panel selection and engagement; case scenario development; and appropriateness ratings by expert panel via two rounds. Specific recommendations were made for children with paediatric and congenital heart disease.

\section{Results}

Recommendations were established for the appropriateness of the selection, characteristics, and insertion technique of intravenous catheters in children with paediatric and congenital heart disease with both univentricular and biventricular physiology.

\section{Conclusion}

miniMAGIC-CHD provides evidence-based criteria for intravenous catheter selection for children with paediatric and congenital heart disease. 


\section{Introduction}

Vascular access in children with paediatric and congenital heart disease (CHD) is a complex process requiring a fundamental understanding of the underlying physiology, disease trajectory, and the procedural skills necessary for vascular access device placement [1]. Traditionally, this decision-making process is inconsistent due to lack of evidence-based criteria for the selection of devices and the techniques of insertion. When clinicians select a vascular access device, a variety of aspects are used to determine which device may be optimal. These include anticipated duration and frequency of use, risk of complications, previous history of vascular access, characteristics of the medications to be administered, and the skill and availability of the operator [2].

When it comes to patients with CHD, many more factors must be considered. An understanding of the anatomy and physiology of the patient, as well as utilization and interpretation of blood sampling based on the catheter site, is imperative. Additionally, preservation of vessels that will need to be accessed for future administration of medications and procedures such as cardiac catheterizations, surgical procedures and possible cardiac transplantation can have life-saving implications [3].

DiPietro and colleagues previously evaluated multi-institutional central venous access device use in patients in North American paediatric cardiac intensive care units [4]. Within the cohort, central venous access device location varied extensively based on physiology and need for cardiac procedure. The most common central venous access device location in patients with a functionally univentricular heart after a Norwood procedure were intracardiac (84\%), followed by umbilical venous catheter (81\%). Following a superior cavopulmonary anastomosis, the most frequent location was intracardiac (56\%), followed by internal jugular vein (43\%), and femoral 
vein (43\%). Following a Fontan procedure, the most common central venous access device location was internal jugular vein $(60 \%)$. The most common locations overall for patients in a cardiac intensive care unit was internal jugular vein followed by intracardiac. As far as complications, the most common location for a thrombus to occur was femoral, and of all central line associated blood stream infections, the largest number came from the femoral location and from peripherally inserted central catheters.

The risk for thrombotic and infectious complications associated with peripherally inserted central catheters were also studied in paediatric inpatients (37\% with cardiac disease) by Noonan and colleagues who demonstrated the rates of central line associated blood stream infections and venous thromboembolism were higher in hospitalized paediatric patients with peripherally inserted central catheters compared to central venous access devices [5].

Using the data available in the literature regarding this population of patients as well as expert consensus, we sought to define recommendations for the use of intravenous catheters for children with CHD and delineate those recommendations between patients with univentricular and biventricular physiology.

\section{Materials and Methods}

The "Michigan Appropriateness Guide for Intravenous Catheters in Pediatrics" (miniMAGIC) was recently developed [2] in accordance with the RAND/UCLA Appropriateness Method [6] to improve the safety and appropriateness of vascular access decision-making for children. Phases of this process included definition of scope and key terms, information synthesis and literature review, expert panel selection and engagement, case scenario development, and lastly, appropriateness ratings by expert panel via two rounds. Indications were classified into 3 levels of appropriateness: 
1. appropriate: panel median score of 7 to 9 , without disagreement;

2. uncertain: panel median score of 4 to 6 or with disagreement regardless of median; and

3. inappropriate: panel median score of 1 to 3 , without disagreement.

Disagreement existed if $\geq 5$ panelists rated in each extreme (1-3 and 7-9) [2].

Following this process, recommendations were made for the use of intravenous catheters in paediatrics. Attention was then turned toward special populations, which included congenital cardiac conditions. Clinical scenarios were divided into two underlying cardiac physiologies: univentricular circulation and biventricular circulation. Scenarios for patients with univentricular physiology were further divided into the stages of palliation, and those with biventricular physiology were further divided by age group. The methods was previously described in detail by Ullman and colleagues We describe here the recommendations developed by miniMAGIC specific to children with congenital heart disease [2].

\section{Results}

In miniMAGIC, a total of 1234 clinical scenarios were reviewed in Round One, which were reduced to 753 scenarios after panel meeting (Round Two). There were 183 CHD-specific scenarios during Round One (22 [12\%] appropriate; 75 [41\%] uncertain; 78 [43\%] inappropriate; 8 [4\%] disagreement), which were reduced to 136 scenarios during the panel meeting (Round Two; 26 [19\%] appropriate; 32 [23\%] uncertain; 76 [56\%] inappropriate; 2 [1\%] disagreement).

For patients with univentricular physiology, miniMAGIC-CHD recommendations were based on the stage of cardiac repair of the patient and the estimated duration of therapy (Figure 1). Lower-extremity (utilizing a peripherally inserted central catheter or femorally inserted central venous access device) and/or umbilical venous catheter (UVC) were rated by the panel as 
appropriate and preferable for patients undergoing stage 1 and 2 palliation, in order to preserve upper-extremity vein patency for stage 2 and 3 palliation [3, 7]. If UVC placement was unsuccessful or has been in place for 14 days or greater, we recommend removal of the UVC and placement of a peripherally inserted central catheter (PICC). Following stage 3 palliation, there was a consensus for appropriateness of upper-extremity access by the panel. For patients at any stage of repair with complex univentricular physiology, the panel discussed the unusual potential necessity to consider alternatives such as trans-hepatic or trans-lumbar access, and recommended such cases have coordinated, interdisciplinary device planning.

In patients with biventricular physiology, miniMAGIC-CHD recommendations were based on the age of the patient and the estimated duration of therapy (Figure 2). Central access via upper-extremity peripherally inserted central catheter or internal jugular vein was preferred based on consensus in order to preserve femoral vein access. This preference was secondary to concern for thrombosis inherent to femoral vascular access devices and the potential need for future femoral venous access for cardiac catheterization procedures $[1,3,4]$. Subclavian venous access was rated uncertain by the panel, due to the limitations of ultrasound use with this placement technique and the variable experience of operators.

Across all scenarios there were two shared recommendations. First, the panel rated totally implanted venous devices as inappropriate in most scenarios regardless of indication because of concerns regarding irreparable vessel damage. Second, the panel rated umbilical catheters as an appropriate access option for neonates because umbilical catheters do not typically result in significant vessel compromise for future procedures.

Lastly, miniMAGIC did not include recommendations surrounding transthoracic intracardiac lines, but recognized placement of these lines can minimize the need for other types 
of vascular access devices, and thereby, minimize the risks associated with percutaneous devices [8]. Children with congenital heart disease may benefit from an intracardiac line placed in the operating room for postoperative monitoring, administration of medications, and sampling of blood. While we support the use of intracardiac lines in this population, it is important to further evaluate formal recommendations for the use of this type of access. Placement of a transthoracic line for the perioperative period may limit the need for other central vascular access, allowing preservation of vessels and minimizing the risk of central line associated blood stream infections.

\section{Discussion}

The miniMAGIC-CHD study is the first study to provide guidance for decisions about vascular access device selection and insertion in paediatric patients with CHD. An understanding of the implications of vascular access device selection in this special population can be lifesaving, by avoiding long-term vessel damage, thrombosis, and occlusion.

Univentricular patients have a unique inherent physiology that changes based on their stage of palliation. Patients who will undergo surgery to create a superior cavopulmonary connections and a total cavopulmonary connections require patent superior and inferior caval veins in order to have optimal outcomes. Debate among clinicians is ongoing as to the optimal catheter location in these patients, due to concern for venous thrombosis [4, 9]. Femoral veins may have a higher incidence of thrombosis, but the impact of an upper extremity thrombus may have greater clinical impact. For example, obstruction to the superior caval vein in patient after a Glenn operation will lead to significant hypoxia and superior vena caval syndrome. In a patient who underwent a Fontan operation, an obstructed superior caval vein may lead to development of a chylothorax, which carries significant potential for consequent morbidity and even mortality $[10]$. 
There is significant benefit to the use of umbilical venous catheters in the neonate [11]. Though not free of complications, umbilical venous catheters provide a reliable source of access while preserving veins in the extremities. Previously published guidelines recommend removal of an umbilical venous catheter by 14 days of use in order to prevent intravascular catheterrelated infections. Thus, we recommend placement of a PICC and removal of a UVC by 14 days, , or if UVC placement was unsuccessful [12]. Additionally, the use of intracardiac lines in the postoperative period is a reasonable alternative to the use of a central line via an extremity, in order to avoid venous obstruction or damage to vessels in an extremity or centrally. These children often require frequent cardiac catheterisations throughout their life for hemodynamic evaluation or intervention, and these catheterisations are ideally performed via vessels in an extremity, usually the femoral vein or the internal jugular vein. In addition, a percentage of this population will undergo cardiac transplantation, and the need for hemodynamic evaluation and myocardial biopsies will be life-long.

Children with biventricular physiology have different risk factors, which are similar to all critically ill paediatric patients. Specifically, access of the subclavian vein can be challenging and uncertain due to variability in operator technique and limitations of ultrasound. These patients also benefit from patent femoral access secondary to the potential need for future procedures or interventions. For example, patients with tetralogy of Fallot or pulmonary atresia may require transcatheter pulmonary valve intervention, and patients receiving a cardiac transplantation will need multiple myocardial biopsies.

A fundamental understanding of all approaches to vascular access is imperative and can be lifesaving in avoiding long-term vessel damage in this special population, who typically require multiple interventions. Interdisciplinary discussion of alternative access devices and 
approaches, such as transhepatic or translumbar, should be considered, particularly in patients who have occluded systemic veins or the need to preserve vessels for future procedures. The feasibility of these alternative routes of access have been previously reported in patients with congenital heart disease. Utilization of transhepatic catheters may be associated with increased rates of complications, such as thrombus formation, catheter dislodgement, and infection, in comparison to traditional catheter routes; however, no long-term complications or increase in infection-related catheter removal have been found [13-17]. Thus, consideration of these alternative access devices and approaches should be considered, though reserved for children with limited availability of other sites [2].

Vessel visualization with ultrasound should be employed whenever achievable to minimize vessel trauma and the number of access attempts. We did not address short term vascular access, where peripheral intravenous catheters (PIVCs) may be appropriate ( 7 days or less). Unless there are specific reasons for a multi-lumen central catheter, a single lumen device should be placed $[18,19]$. Totally implantable venous devices do not typically have a role in the congenital cardiac population.

We did not make recommendations for short term use of venous access, and it may be reasonable to avoid central access in patients who require only peripherally compatible infusions to minimize infection and thrombosis risk. Future studies should help delineate timing and risk of thrombosis/infection inherent to specific catheters in this population.

The uncertainty of the risk and benefit profile of many of these decisions portends the importance of a multidisciplinary approach, including cardiologists, intensivists, surgeons, nurses, anaesthesiologists, radiologists, and other allied professionals. However, these 
recommendations can serve as a guide to the approach to vascular access in this complex patient population. 


\section{Acknowledgements}

Funding Source: miniMAGIC was supported by grants from the Association for Vascular Access Foundation, Griffith University, and the University of Michigan. No funding was provided directly for the development of this publication.

\section{Financial Disclosure:}

Amanda Ullman reports fellowships and grants by the National Health and Medical Research Council (NHMRC), employment by Griffith University, grants by the Children's Hospital Foundation, the Royal Brisbane and Women's Hospital Foundation, Emergency Medicine Foundation, and the Australian College of Critical Care Nursing; and investigator-initiated research grants and speaker fees provided to Griffith University from 3M Medical, Angiodynamics, Becton Dickinson and Cardinal Health (unrelated to the current project).

The other authors have no disclosures.

\section{Potential Conflict of Interest:}

Amanda Ullman reports investigator-initiated research grants and speaker fees provided to Griffith University from vascular access product manufacturers (3M Medical, Becton Dickinson, Cardinal Health), unrelated to the current project. Ms Pitts reports employment by B. Braun Medical Inc. and St Joseph's Children's Hospital, Tampa, Florida, previous employment and stockholder status with AngioDynamics, and board membership of Navi Medical Technologies.

The other authors have no conflicts of interest to disclose. 


\section{References}

1. Aiyagari, R., D.S. Cooper, and J.P. Jacobs, Vascular Access in Children With Congenital Heart Defects. Pediatrics, 2020. 145(Suppl 3): p. S294-s295.

2. Ullman, A.J., et al., The Michigan Appropriateness Guide for Intravenous Catheters in Pediatrics: miniMAGIC. Pediatrics, 2020. 145(Suppl 3): p. S269-s284.

3. Aiyagari, R., et al., Central venous catheter-associated complications in infants with single ventricle: comparison of umbilical and femoral venous access routes. Pediatr Crit Care Med, 2012. 13(5): p. 549-53.

4. DiPietro, L.M., et al., Central Venous Catheter Utilization and Complications in the Pediatric Cardiac ICU: A Report From the Pediatric Cardiac Critical Care Consortium (PC4). Pediatr Crit Care Med, 2020. 21(8): p. 729-737.

5. Noonan, P.J., et al., Comparison of Complication Rates of Central Venous Catheters Versus Peripherally Inserted Central Venous Catheters in Pediatric Patients. Pediatr Crit Care Med, 2018. 19(12): p. 1097-1105.

6. $\quad$ Fitch, K., et al., The RAND/UCLA appropriateness method user's manual. 2001, Rand Corp Santa Monica CA.

7. Jacobs, J.P., et al., The Society of Thoracic Surgeons Congenital Heart Surgery Database Mortality Risk Model: Part 2-Clinical Application. Ann Thorac Surg, 2015. 100(3): p. 1063-8; discussion 1068-70.

8. Kumar, T.K.S., et al., Optimum position of transthoracic intracardiac line following cardiac surgery. Interact Cardiovasc Thorac Surg, 2017. 25(6): p. 883-886.

9. Miller, J.W., et al., Upper body central venous catheters in pediatric cardiac surgery. Paediatr Anaesth, 2013. 23(11): p. 980-8.

10. Perry, T., et al., Non-surgical Risk Factors for the Development of Chylothorax in Children after Cardiac Surgery-Does Fluid Matter? Pediatr Cardiol, 2020. 41(1): p. 194-200.

11. Stein, M.L., et al., Complications of Transthoracic Intracardiac and Central Venous Lines in Neonates Undergoing Cardiac Surgery. Pediatr Cardiol, 2019. 40(4): p. 733-737.

12. O'Grady, N.P., et al., Guidelines for the prevention of intravascular catheter-related infections. Clin Infect Dis, 2011. 52(9): p. e162-93.

13. Marshall, A.M., et al., Traditional Long-Term Central Venous Catheters Versus Transhepatic Venous Catheters in Infants and Young Children. Pediatr Crit Care Med, 2017. 18(10): p. 944-948.

14. Boe, B.A., et al., Transhepatic Central Venous Catheters in Pediatric Patients With Congenital Heart Disease. Pediatr Crit Care Med, 2015. 16(8): p. 726-32.

15. Mortell, A., et al., Transhepatic central venous catheter for long-term access in paediatric patients. J Pediatr Surg, 2008. 43(2): p. 344-7.

16. Qureshi, A.M., et al., Transhepatic Broviac catheter placement for long-term central venous access in critically ill children with complex congenital heart disease. Pediatr Crit Care Med, 2007. 8(3): p. 248-53.

17. Inston, N., et al., Options for end stage vascular access: Translumbar catheter, arterial-arterial access or right atrial graft? J Vasc Access, 2020. 21(1): p. 7-18.

18. Bozaan, D., et al., Less Lumens-Less Risk: A Pilot Intervention to Increase the Use of Single-Lumen Peripherally Inserted Central Catheters. J Hosp Med, 2019. 14(1): p. 42-46.

19. Chopra, V., et al., The Michigan Appropriateness Guide for Intravenous Catheters (MAGIC): Results From a Multispecialty Panel Using the RAND/UCLA Appropriateness Method. Ann Intern Med, 2015. 163(6 Suppl): p. S1-40. 
Page 13 of 16 


\section{Figure legends}

Figure 1. miniMAGIC recommendations for congenital cardiac conditions in paediatric patients with functionally univentricular physiology requiring venous access for greater than 7 days. Fem, femoral; Jug, jugular; Low, lower body; PIVC, peripheral intravenous catheter; PICC, peripherally inserted central catheter; NTCVAD, non-tunnelled central venous access device; Sub; subclavian; TcCVAD, tunnelled, cuffed central venous access device; TIVD, totally implanted venous device; TncCVAD, tunnelled, non-cuffed central venous access device; Up, upper body.

Figure 2. miniMAGIC recommendations for congenital cardiac conditions in paediatric patients with biventricular circulation requiring venous access for greater than 7 days. * Disagreement. Fem, femoral; Jug, jugular; Low, lower body; PIVC, peripheral intravenous catheter; PICC, peripherally inserted central catheter; NTCVAD, non-tunnelled central venous access device; Sub; subclavian; TcCVAD, tunnelled, cuffed central venous access device; TIVD, totally implanted venous device; TncCVAD, tunnelled, non-cuffed central venous access device; Up, upper body. 


\section{Figures}

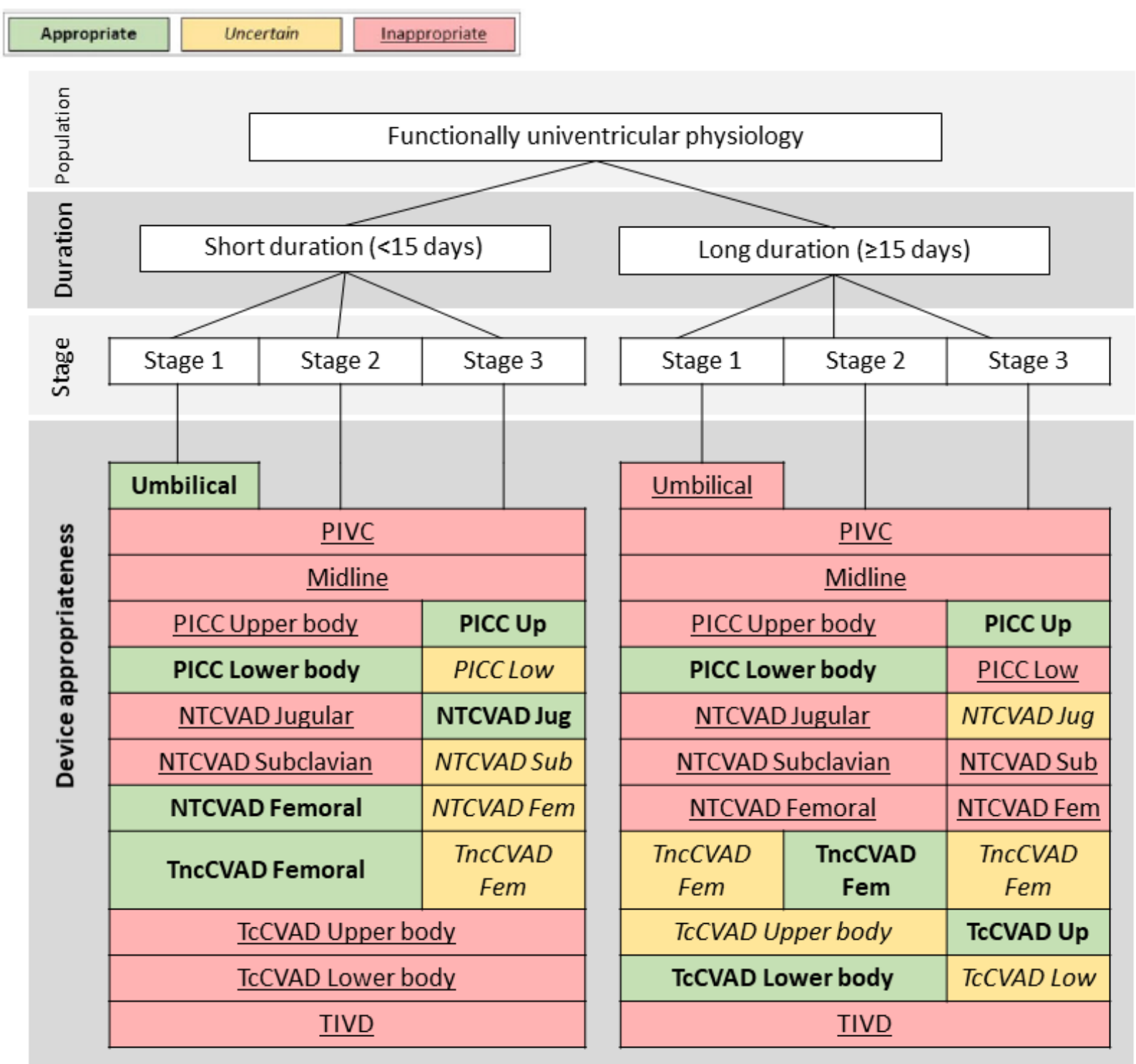

Figure 1. 


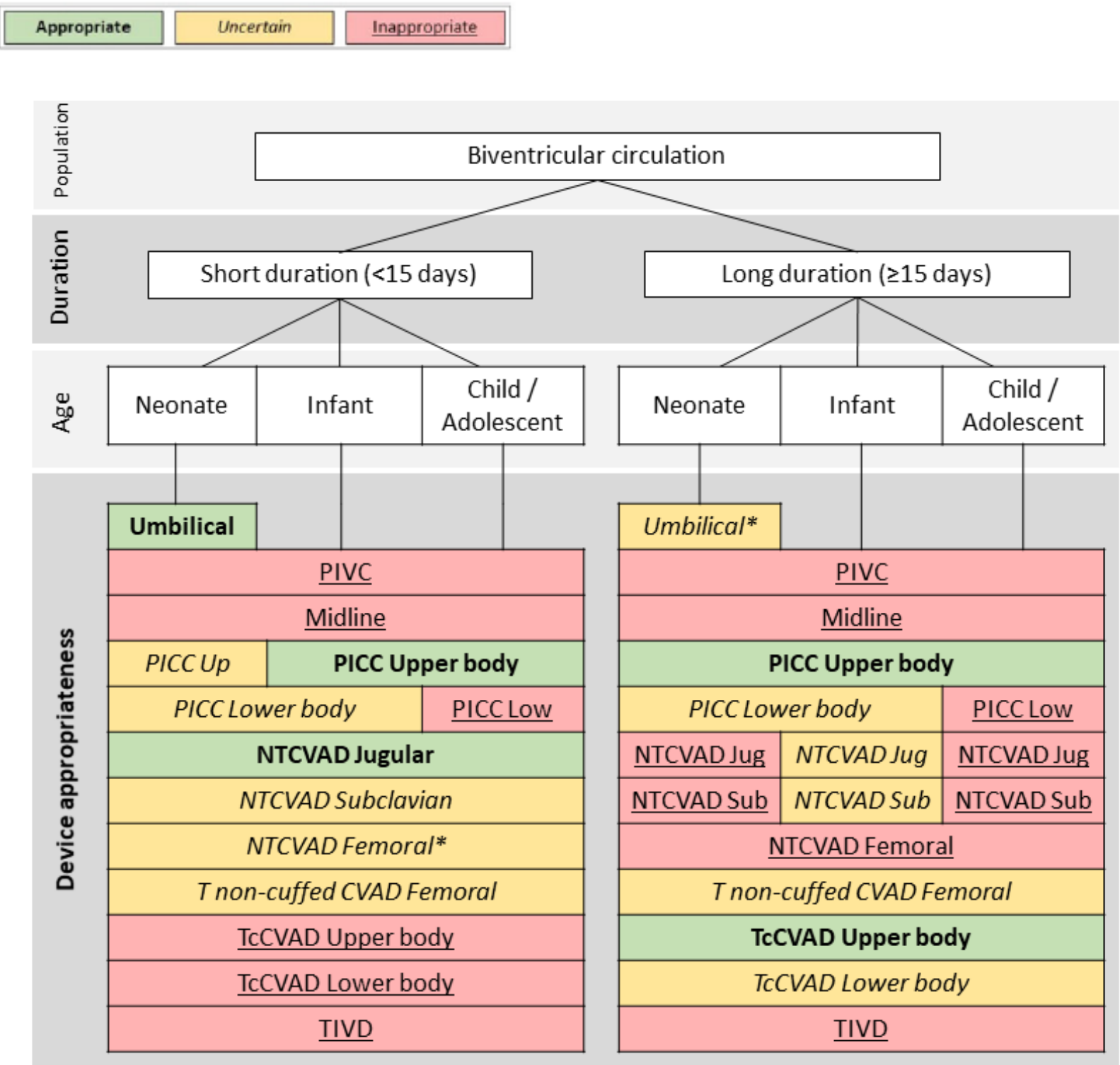

Figure 2. 